\title{
A practical multiscale approach for optimization of structural damping
}

\author{
Andreassen, Erik; Jensen, Jakob Søndergaard
}

Published in:

Structural and Multidisciplinary Optimization

Link to article, DOI:

$10.1007 / \mathrm{s} 00158-015-1326-9$

Publication date:

2016

Document Version

Peer reviewed version

Link back to DTU Orbit

Citation (APA):

Andreassen, E., \& Jensen, J. S. (2016). A practical multiscale approach for optimization of structural damping. Structural and Multidisciplinary Optimization, 53(2), 215-224. https://doi.org/10.1007/s00158-015-1326-9

\section{General rights}

Copyright and moral rights for the publications made accessible in the public portal are retained by the authors and/or other copyright owners and it is a condition of accessing publications that users recognise and abide by the legal requirements associated with these rights.

- Users may download and print one copy of any publication from the public portal for the purpose of private study or research.

- You may not further distribute the material or use it for any profit-making activity or commercial gain

- You may freely distribute the URL identifying the publication in the public portal

If you believe that this document breaches copyright please contact us providing details, and we will remove access to the work immediately and investigate your claim. 
Noname manuscript No.

(will be inserted by the editor)

\title{
A practical multiscale approach for optimization of structural damping
}

\author{
Erik Andreassen · Jakob Søndergaard Jensen
}

Received: date / Accepted: date

\begin{abstract}
A simple and practical multiscale approach suitable for topology optimization of structural damping in a component ready for additive manufacturing is presented. The approach consists of two steps: First, the homogenized loss factor of a two-phase material is maximized. This is done in order to obtain a range of isotropic microstructures that have a connected stiff material phase. Second, the structural damping of the component is maximized using material interpolations based on the homogenized properties of the microstructures. In order to achieve convergence towards a discrete set of material phases in the macroscopic problem, a material interpolation that favors values close to the predefined material densities is introduced.
\end{abstract}

Keywords damping $\cdot$ microstructure $\cdot$ topology optimization $\cdot$ homogenization $\cdot$ multiscale $\cdot$ additive manufacturing

\section{Introduction}

The aim of this paper is to propose a practical multiscale formulation for the maximization of a component's structural damping, which yields a component that can be manufactured by printing the stiff phase (typically a metal), e. g. using the additive manufacturing method selective laser sintering, and then be used as a mold for the soft phase.

The basic idea of much of the multiscale work in the field of topology optimization is that it is possible to map intermediate densities to microstructures with properties found by solving a set of local microstructure optimization problems. Rodrigues et al (2002) were among the first to suggest such an iterative approach, based on earlier works by among others Bendsøe et al (1994), and Theocaris and Stavroulakis (1999). Coelho et al (2008) extended the approach to three-dimensional problems, and even applied it to bone replacement designs (see e.g. Coelho et al 2011). The approach is relatively straightforward to implement for minimization of compliance, and it has been shown to work well. It should also be mentioned that Barbarosie and Toader (2012) have worked on extending the approach to include shape optimization on the unit cell level.

One drawback with the method is that it is not guaranteed to converge towards a manufacturable design. The fact that the microstructures are not necessarily connected can, at least for minimum compliance problems, be amended by post-processing (shifting the base unit cells until they match). However, this would be rather time-consuming. Furthermore, it is uncertain whether the approach would work equally well for more complex problems.

An alternative approach is to predefine regions which should have the same material, and then optimize the material structure in each of these regions for a macroscopic response (as done by Andreasen and Sigmund (2012), and with a full solution of the macroscopic problem by Alexandersen and Lazarov (2015)).

This work was funded by the Danish Research Agency through the innovation consortium F.MAT.

E. Andreassen

Department of Mechanical Engineering, Technical University of Denmark,

E-mail: erand@mek.dtu.dk

J. S. Jensen

Department of Electrical Engineering, Technical University of Denmark, Nils Koppels Allé, Building 404, 2800 Lyngby, Denmark 
Thus, simplifying the matter and making it easier to generalize to problems involving multiphysics, but also restricting the design space.

A third approach, suggested by among others Theocaris and Stavroulakis (1999), is to base the material interpolation on an a priori defined material library. A manufacturable two-dimensional (2D) bone replacement prototype was achieved by Khanoki and Pasini (2012) using a precomputed interpolation for a simple orthotropic microstructure in the optimization.

In the following we will show how this third approach can be applied in a multiscale optimization of the structural damping of a component build from two material phases. First, the homogenized loss factor is maximized by using an approach similar to the one described in (Andreasen et al 2014). This is done in order to obtain a range of isotropic microstructures with a connected stiff material phase, which can be used in a multi-material optimization problem. In order to achieve convergence towards a discrete set of material phases in the macroscopic problem, a material interpolation that favors values close to the predefined material densities is introduced.

An alternative way to achieve a design with discrete microstructures, would be to use an approach similar to what is done in optimization of fiber reinforced composites with a discrete set of fiber angles (see description of discrete material optimization (DMO) by Stegmann and Lund 2005). The implementation would require multiple design fields, and this is not necessary with the formulation presented here. However, a DMO approach would probably be useful if the presented method should be extended to anisotropic material structures.

The structure of the paper is such that most of the theory is presented in Section 2, where first the macroscopic problem is presented in Section 2.1, which also includes a justification for the multiscale approach, and then the microscopic problem is presented in Section 2.2. Thereafter, Section 3 follows with an example illustrating how a realistic material interpolation for the macroscopic problem can be obtained and how this influences the macroscopic design. The manufacturability of the design is not considered in Section 3.

Results for manufacturable microstructures are presented in Section 4. Then, with basis in the obtained results, Section 5 presents the material interpolation that makes the macroscopic problem converge to a discrete set of densities. The results for the macroscopic problem are presented in Section 6, before the conclusion in Section 7.

\section{Theory}

\subsection{Macroscopic optimization problem}

The basic formulation of the macroscopic problem is similar to the one presented by Kim et al (2013), who use topology optimization to find the best layout of damping treatments on a cylindrical shell structure. Other similar formulations have been suggested by Ling et al (2011); El-Sabbagh and Baz (2014) for damping treatments of plane plate structures, but where the final designs contain large regions with intermediate densities.

The problem considered here is that of maximizing a component's structural damping for a range of eigenfrequencies by optimizing the distribution of the material. The macroscopic optimization problem is:

$$
\begin{array}{lll}
\min _{\varphi}: & \max \left(-\log \zeta_{i}\right), & i=1, \ldots, n \\
\text { s. t.: } & \left(\mathbf{K}-\omega^{2} \mathbf{M}\right) \mathbf{U}=\mathbf{0}, \quad & \text { Eigenvalue problem } \\
& \operatorname{Re}\left(\omega_{1}\right) \geq \omega_{0}, \quad \text { Constraint on first eigenvalue } \\
& 0 \leq \varphi_{e} \leq 1, \quad e=1, \ldots, m & \text { Macroscopic design variables }
\end{array}
$$

where the objective is to maximize the smallest of the structural damping ratios $\zeta_{i}$ for the $n$ first eigenfrequencies. $\zeta_{i}$ can be computed from the complex eigenvalue $\omega_{i}$ as

$$
\zeta_{i}=\sqrt{\frac{1}{1+\left(\operatorname{Re}\left(\omega_{i}\right) / \operatorname{Im}\left(\omega_{i}\right)\right)^{2}}} .
$$

The eigenvalue problem is a standard finite element eigenvalue problem, where the component's stiffness and mass matrix, $\mathbf{K}$ and $\mathbf{M}$, are assembled in the standard manner by summing over the elements. Remark, though, $\mathbf{K}$ will be complex since the component's damping is modelled as structural damping, where the elasticity tensor has both a real and imaginary part. 
Table 1: Material properties for the stiff material phase and two different soft material phases. The material phases are isotropic, and, thus, the material properties are uniquely given by Young's modulus $E=E^{\prime}(1+i \eta)$ and Poisson's ratio $v$, but for the sake of clarity $K^{\prime}$ and $G^{\prime}$ are also stated.

\begin{tabular}{ccccccc}
\hline & $\rho$ & $E^{\prime}$ & $v$ & $G^{\prime}$ & $K^{\prime}$ & $\eta$ \\
Material & {$\left[\mathrm{kg} / \mathrm{m}^{3}\right]$} & {$[\mathrm{GPa}]$} & {$[-]$} & {$[\mathrm{GPa}]$} & {$[\mathrm{GPa}]$} & {$[-]$} \\
\hline Titanium & 4500 & 100 & 0.3 & 38.46 & 83.33 & 0.001 \\
S1 & 1000 & 2 & 0.4 & 0.71 & 3.33 & 0.05 \\
$\mathrm{~S} 2$ & 1000 & 0.05 & 0.495 & 0.017 & 1.67 & 0.5 \\
\hline
\end{tabular}

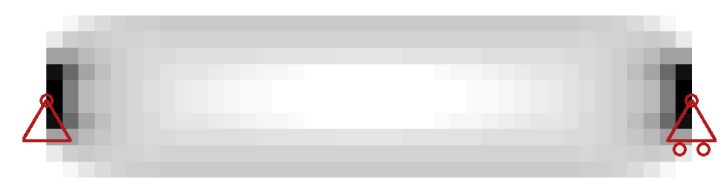

Fig. 1: Result obtained using the optimization formulation in Eq. (1) with a linear interpolation between the material properties of S1 (white) and titanium (black). The design mesh consists of $40 \times 10$ elements, and the finite element mesh of $80 \times 20$ elements.

The constraint on the lowest eigenvalue is present to assure a minimum stiffness of the component, which would be required in most applications. Without this constraint, the optimal design would be to use only the softest material. Note, filtering of the design variables is not used for this macroscopic problem.

The key point of the multiscale approach is to base the material interpolation in the macroscopic problem on the homogenized material properties of corresponding microstructures. That is, the design variable value for an element, corresponding to a normalized density, can be mapped to a microstructure with material properties found from homogenization - also when the design variable is in-between 0 and 1.

There are two main reasons to use a multiscale approach. First, and most important to us, to assure the stiff phase can be manufactured in one piece using additive manufacturing and infused with the soft material. Second, because resolving the macroscopic problem down to the manufacturing precision can be extremely computationally expensive, especially since it involves solving an eigenvalue problem.

\subsubsection{Simply supported beam}

The macroscopic design problem considered in this paper is that of a $2 \mathrm{D}$ simply supported beam with dimensions $200 \mathrm{~mm} \times 50 \mathrm{~mm}$. The dimensions are chosen based on the fact that few additive manufacturing machines have a build chamber where the largest dimension is more than $200 \mathrm{~mm}$. Furthermore, assume the minimum feature size (wall thickness) is $1 / 4 \mathrm{~mm}$. To resolve the manufacturing precision of this simple 2D problem, $800 \times 200$ elements would be necessary. However, if each element was mapped to a microstructure with a unit cell size of $5 \times 5 \mathrm{~mm}$, a mere $40 \times 10$ design elements are necessary.

Solving the optimization problem in Eq. (1) on this coarse design variable mesh with a linear interpolation between the material properties of a stiff phase (titanium, see Tab. 1) and a soft phase ( $\mathrm{S} 1$ in Tab. $1)$, with $\omega_{0}=80 \% \operatorname{Re}\left(\omega_{1}^{\mathrm{t}}\right)$, where $\omega_{1}^{\mathrm{t}}$ is the first eigenfrequency of pure titanium, results in the design in Fig. 1. Even though we use a design mesh of $40 \times 10$ elements, the finite element mesh consists of $80 \times 20$ elements. Partly, because we want to make sure the physics is captured properly, and partly, because it makes the comparison with one of the examples in Section 5, where a finer design mesh is used, more fair.

The design consists mostly of intermediate densities (gray). However, this is not only due to the coarse mesh, but also because the material properties are interpolated linearly, which is not realistic. In order to obtain a more realistic interpolation, it is necessary to consider the design of the microstructure.

\subsection{Microscopic optimization problem}

As mentioned, a practical approach to multiscale optimization is to optimize the properties of the microstructure first, and then use these optimized microstructures as material phases to interpolate between with a multi-material interpolation scheme. With this approach the connectivity of the graded microstructure can be assured a priori, which is an important consideration and not trivial to achieve. Furthermore, the computational burden is very low compared to a coupled multiscale optimization problem. The drawback is that the 


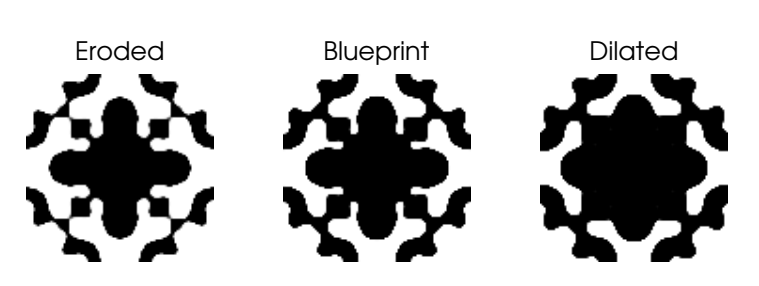

Fig. 2: Illustration of relation between blueprint unit cell of the microstructure (to be manufactured) and the eroded and dilated versions, which are also included in the optimization problem.

solution space is more limited. For the problem presented here, where the focus is on isotropic microstructures, this is not a big issue. If arbitrarily anisotropic microstructures should be allowed, the approach might not be suitable. However, one would still need to require connectivity (with a minimum length scale) between the cells in both the horizontal and vertical direction, which for the considered problem would highly limit the benefits of including anisotropy.

In order to obtain manufacturable microstructures with a minimum feature size, a robust formulation Wang et al (2011) is used. This means that in addition to the blueprint microstructure (the one you want to manufacture), an eroded and a dilated version of the blueprint are also included in the optimization. The difference between the three design is illustrated in Fig. 2. The three designs are included in the optimization formulation using a min-max approach in which the homogenized properties are computed for all three realizations.

In Andreasen et al (2014), a formulation to obtain microstructures with a maximized bulk loss modulus is presented. This results in microstructures that can have very different loss factors in bulk and shear. Therefore, a slightly modified approach is used here, where the goal is to maximize the smallest of the bulk and shear loss factor. Furthermore, the stiffness constraint is on the shear modulus instead of the bulk modulus. For a 2D unit cell, this can be formulated as:

$$
\begin{array}{lll}
\min _{\boldsymbol{\varphi}^{\text {micro }}}: & \max \left(\eta_{G}^{*}, \eta_{K}^{*}\right) & \text { Loss factors (also dilated and eroded design) } \\
\text { s.t.: } & \mathbf{K U}=\mathbf{F}, & \text { Cell equilibrium equations } \\
& \operatorname{Re}\left(G^{*}\right) \geq a \operatorname{Re}\left(G_{\text {stiff }}\right), & \text { Shear storage modulus constraint } \\
& \frac{\sum_{i, j}\left(\operatorname{Re}\left(C_{i j}^{\text {iso }}-C_{i j}^{*}\right)\right)^{2}}{\left(\operatorname{Re}\left(C_{11}^{\text {iso }}\right)\right)^{2}} \leq \varepsilon, \quad i, j=1, \ldots, 3 & \text { Isotropy constraint (real part) } \\
& \frac{\sum_{i, j}\left(\operatorname{Im}\left(C_{i j}^{\text {iso }}-C_{i j}^{*}\right)\right)^{2}}{\left(\operatorname{Im}\left(C_{11}^{\text {iso })}\right)\right)^{2}} \leq \varepsilon, \quad i, j=1, \ldots, 3 & \\
& \text { Isotropy constraint (imaginary part) } \\
& \mathbf{K}_{\sigma} \mathbf{U}_{\sigma}=\mathbf{F}_{\sigma}, & \text { Cell equilibrium equations (conductivity) } \\
\sigma^{*} \geq b \sigma_{\text {stiff }}, & \text { Conductivity constraint on stiff phase } \\
& 0 \leq \varphi_{e}^{\text {micro }} \leq 1, \quad e=1, \ldots, N & \text { Local design variables }
\end{array}
$$

where $\eta_{G}^{*}$ and $\eta_{K}^{*}$ are the homogenized shear and bulk modulus loss factors, respectively. $G^{*}$ is the homogenized shear modulus, and $G_{\text {stiff }}$ is the shear modulus of the stiffest material phase. Isotropy is enforced on both the real and imaginary part of the homogenized constitutive matrix with entries $C_{i j}^{*}\left(\varepsilon=10^{-5}\right.$ and $C_{i j}^{\text {iso }}$ is computed based on the average of $C_{11}^{*}$ and $C_{22}^{*}$, and $C_{12}^{*}$ ). The conductivity constraint is there to assure a connected stiff phase, therefore the homogenized conductivity is computed by setting the conductivity of the soft phase $\sigma_{\text {soft }}=10^{-6} \sigma_{\text {stiff. }}$. The homogenized material properties are found by solving the equilibrium equations on the unit cell and computing the homogenized tensors (Guedes and Kikuchi 1990; Hassani and Hinton 1998). A Matlab implementation showing the details of how this is done can be found in (Andreassen and Andreasen 2014).

Note, both the shear modulus constraint and the conductivity constraint are on the eroded design. With this formulation, the resulting microstructure has a connected stiff phase, and has a minimum length scale on both the soft and stiff phase. Furthermore, if the obtained shear and bulk loss factor are equal, the material phase can be fully described using only three parameters: $G^{\prime}, K^{\prime}$, and $\eta$, which can be interpolated using the same design variable. The material interpolation in the microscopic elasticity problem is linear, and for the conductivity problem, a penalization factor of 3 is used. The macroscopic and microscopic optimization are weakly coupled in the sense that results from the microscopic optimization problem is used in the macroscopic problem. 


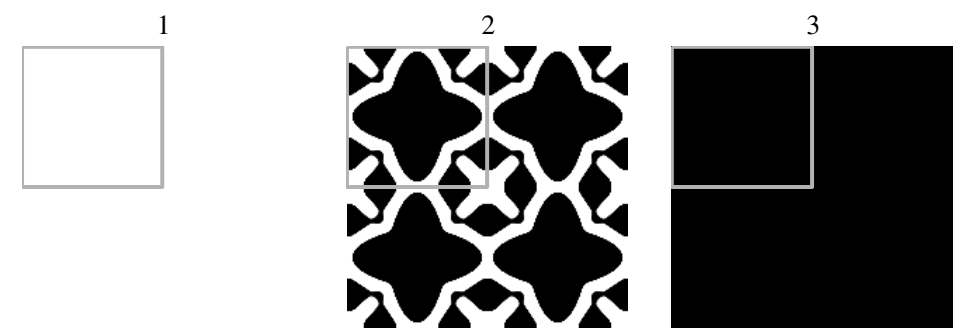

(a) (a)

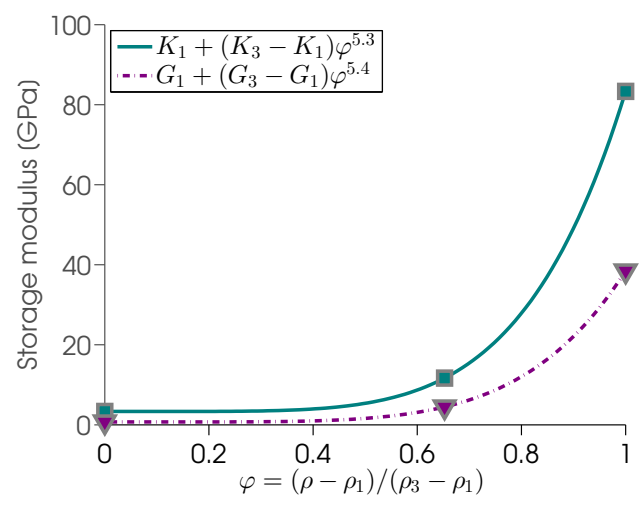

(b) (b)

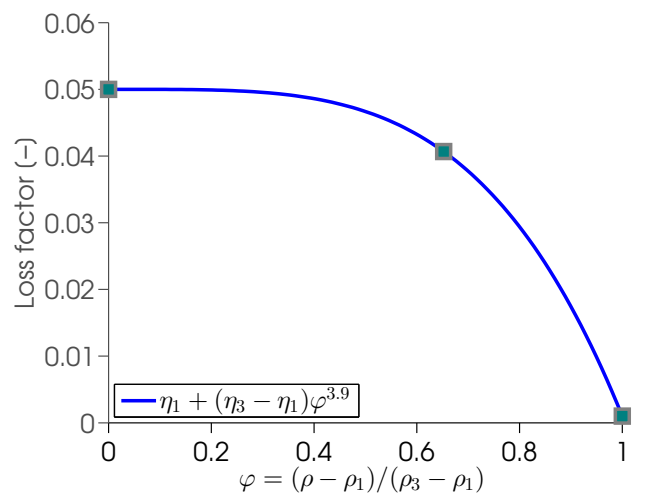

(c) (c)

Fig. 3: (a) Microstructure 2 is obtained with the optimization formulation in Eq. (3) with $\mathrm{S} 1$ as the soft phase (microstructure 1) and no robustness requirement or conductivity constraint. (b-c) Power law interpolation between pure soft/stiff phase and the microstructure in (a). The shear and bulk loss factor are the same.

With the microscopic optimization problem presented here, it is easy to obtain 2D microstructures that fulfill the manufacturing requirements. The formulation is based on the one presented in Andreasen et al (2014), where it is shown that even though the formulation does not guarantee connected stiff regions, it seems to favor open cells (also for three-dimensional microstructures) as long as the conductivity constraint is not too low. In our experience, the robust formulation will further favor open cells (small features are not allowed in the blueprint design). The Method of Moving Asymptotes (MMA) is used for all the numerical optimizations (Svanberg 1987).

\section{Realistic material interpolations}

A simple way to obtain a material interpolation between titanium and S1, is to use the formulation in Eq. (3) to find a microstructure with an intermediate density, and use the microstructures homogenized properties to create a power law interpolation between the material properties of S1 and titanium. In this section we want to obtain a material interpolation with the highest possible loss factor for the intermediate microstructure, and, therefore, the requirements necessary for manufacturing (robustness and conductivity of stiff phase) are disregarded.

For $a=0.1$ (the shear modulus constraint) the resulting microstructure is shown in Fig. 3(a) and denoted microstructure 2 (microstructure 1 is pure $\mathrm{S} 1$, and microstructure 2 is pure titanium). White is used to indicate the soft material phase, while black is used for the stiff material phase (titanium). The material properties of the obtained microstructure are plotted together with the material properties of S1 and titanium in Fig. 3(b-c), together with power law fitted functions, which can be used as material interpolation functions in the macroscopic problem. The horizontal axis show the normalized density, computed as

$$
\varphi=\frac{\rho-\rho_{1}}{\rho_{3}-\rho_{1}}
$$

where $\rho_{1}$ is the density of the softest (and lightest) microstructure, $\rho_{3}$ is the density of the stiffest (and heaviest) microstructure, and $\rho \in\left[\rho_{1}, \rho_{3}\right]$. 


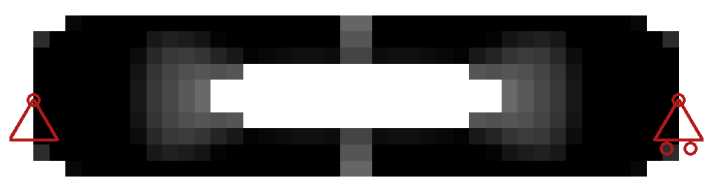

(a) (a)

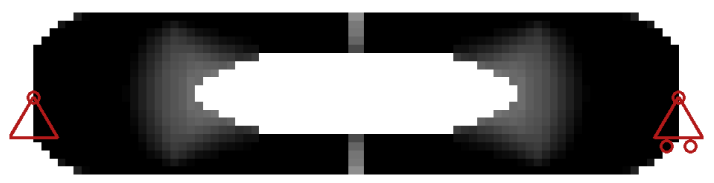

(b) (b)

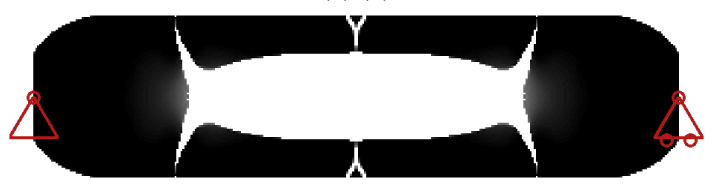

(c) (c)

Fig. 4: Result obtained using the optimization formulation in Eq. (1) with the power law interpolations stated in Fig. 3 between the material properties of S1 (white) and titanium (stiff). In (a) the design mesh consists of $40 \times 10$ elements, while in (b) it consists of $80 \times 20$ elements. In both (a) and (b) the finite element mesh consists of $80 \times 20$ elements. In (c) both the design and finite element mesh consist of $320 \times 80$ elements.

Rerunning the macroscopic problem with the power law interpolations depicted in Fig. 3(b-c), results in the designs in Fig. 4, where also two designs obtained with finer design meshes are depicted.

With this more realistic interpolation, the design contains a much lower fraction of intermediate densities. However, there are still intermediate densities present - also when the resolution is increased. There is no guarantee that all points in the interpolation can be mapped to microstructures, and this could possibly to some degree explain the intermediate densities in the designs, but the results still indicate that even with a realistic interpolation a very fine discretization can be necessary to obtain a design without intermediate densities. But it also indicates that there is little benefit, for the macroscopic objective in mixing the two material phases on a micro-level. Thus, the two main reasons for creating the microstructure are: 1) It is important from a manufacturing point of view (e.g. to achieve connected stiff phase), 2) Computational resources limit the macroscopic problem to a very coarse resolution.

\section{Microstructure results}

In this section results for manufacturable microstructures with a stiff phase are presented. The microscopic formulation in Eq. (3) is used to maximize the loss factor of three connected microstructures consisting of titanium and S1, and titanium and S2. Where S2 is a soft phase much closer to a rubber material than S1. The material properties are given in Tab. 1.

A plane strain formulation is used, such that material phase S2, which has a Poisson's ratio close to 0.5, will be nearly incompressible. In order to prevent volumetric locking, the B-Bar method (Hughes 1980), which for rectilinear elements is equivalent to selective reduced integration, is used. However, for the microscopic homogenization problem, where S2 is used, locking does not seem to be an issue. The reason for this might be that the constitutive tensor is also reflected in the load. Therefore, almost identical results can be obtained without using selective reduced integration.

First, the microscopic formulation in Eq. (3) is used to maximize the loss factor of three connected microstructures consisting of titanium and S1, and titanium and S2.

For both cases, the loss factor is maximized with three different values $a$ for the stiffness constraint. The conductivity constraint value is set to $b=0.05$, and the robust formulation with threshold parameters 0.4 (dilated), 0.5 (blueprint), and 0.6 (eroded). $b$ should be chosen relatively low, such that it does not restrict the design too much, but not so small that the eroded design contains floating islands. We have found 0.05 to be a good compromise A filter radius of 0.07 times the unit cell width, have been used for all designs. A higher conductivity constraint would typically give larger stiff material phase feature sizes, and a larger filter radius 


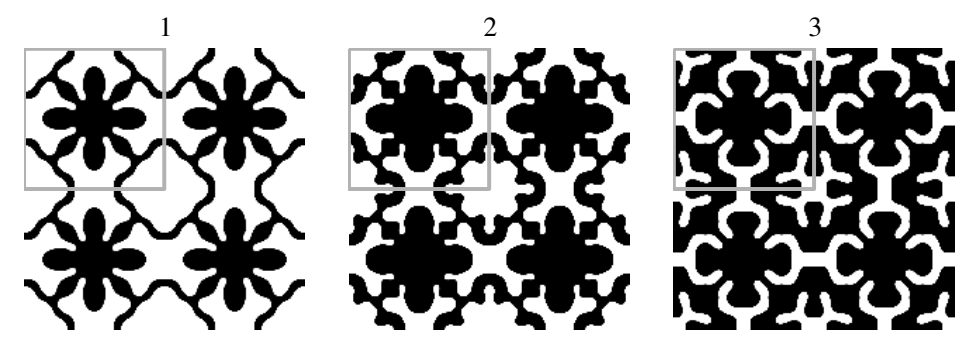

(a) (a)

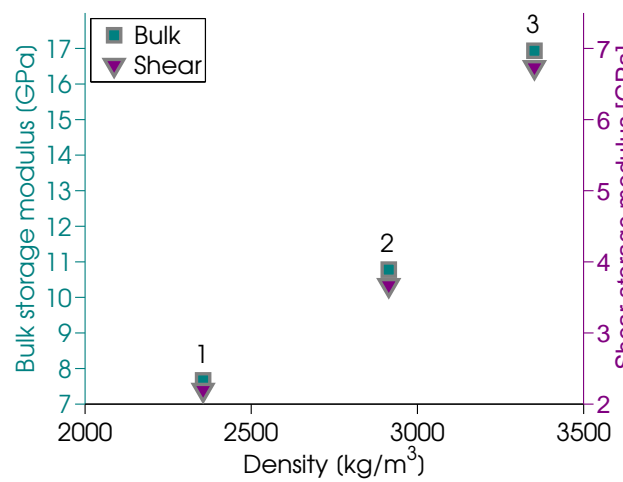

(b) (b)

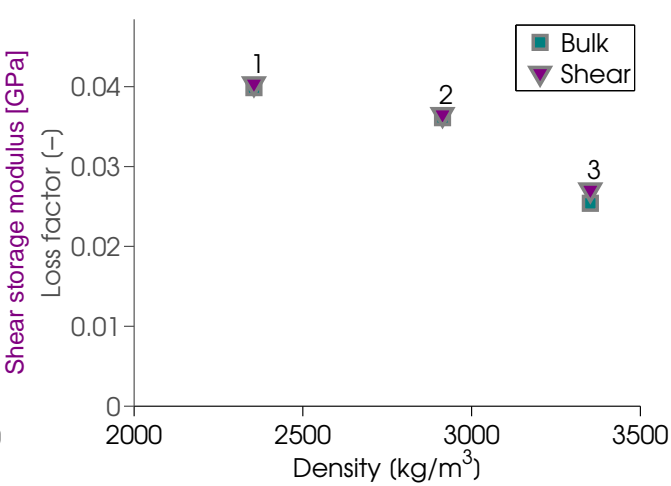

(c) (c)

Fig. 5: Results obtained using the optimization formulation in Eq. (3) with S1 as the soft phase. (a) The three microstructures, and (b-c) their corresponding properties.

Table 2: Material properties for the titanium-S1 and the titanium-S2 microstructures in Fig. 5 and 6.

\begin{tabular}{cccccc}
\hline Material & $\begin{array}{c}\rho \\
{\left[\mathrm{kg} / \mathrm{m}^{3}\right]}\end{array}$ & $\begin{array}{c}G^{\prime} \\
{[\mathrm{GPa}]}\end{array}$ & $\begin{array}{c}K^{\prime} \\
{[\mathrm{GPa}]}\end{array}$ & $\begin{array}{c}\eta_{G} \\
{[-]}\end{array}$ & $\begin{array}{c}\eta_{K} \\
{[-]}\end{array}$ \\
\hline S1-T (1) & 2355 & 2.2 & 7.7 & 0.040 & 0.040 \\
S1-T (2) & 2914 & 3.7 & 10.8 & 0.037 & 0.036 \\
S1-T (3) & 3352 & 6.7 & 16.9 & 0.027 & 0.025 \\
\hline S2-T (1) & 2798 & 1.0 & 4.2 & 0.31 & 0.45 \\
S2-T (2) & 3349 & 3.7 & 6.1 & 0.25 & 0.40 \\
S2-T (3) & 3947 & 8.1 & 12.6 & 0.16 & 0.31 \\
\hline
\end{tabular}

(or wider $\eta$-values) will increase the minimum feature size. A 45 degree symmetry has been enforced in all designs - this makes it easier to fulfill the isotropy constraints and do not, based on numerical experiments, affect the performance.

The resulting microstructures when using S1 are shown in Fig. 5(a). The corresponding homogenized material properties (evaluated from the thresholded designs) are plotted in Fig. 5(b-c). The stiffness of the microstructures follow a power law with a low exponent, and the loss factors seem to follow a similar power law, just with the opposite sign. Furthermore, note that the bulk storage modulus is always larger than the shear, even though the constraint is on the shear storage modulus.

The resulting microstructures when using S2 are shown in Fig. 6(a), and the corresponding homogenized material properties are plotted in Fig. 6(b-c). Due to the high bulk modulus of S2, it is possible to obtain four connected microstructures with a high loss factor. Even though the shear loss factor is lower than the bulk loss factor for all the microstructures, the S2 microstructures seem to have a good compromise between damping and stiffness, but are denser and all the microstructures have a slightly lower bulk modulus than the corresponding S1 microstructures.

The homogenized material properties of both the titanium-S1 and the titanium-S2 microstructures are summarized in Tab. 2. 


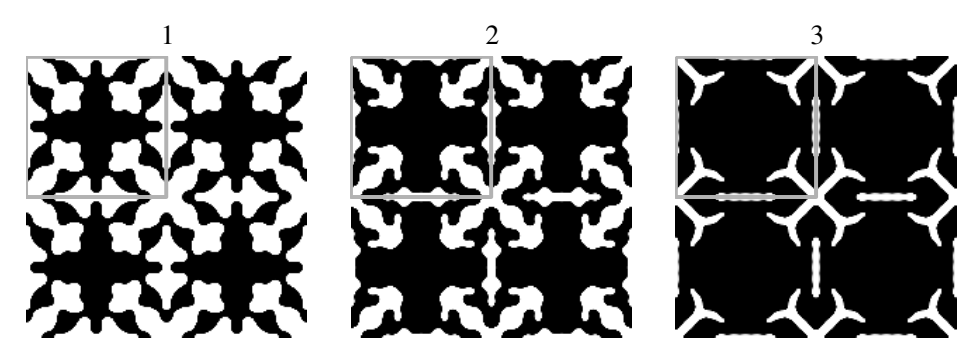

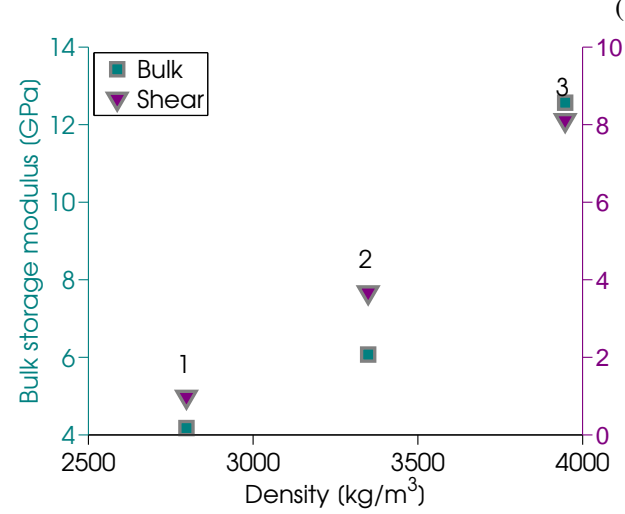

(b) (b) (a) (a) 


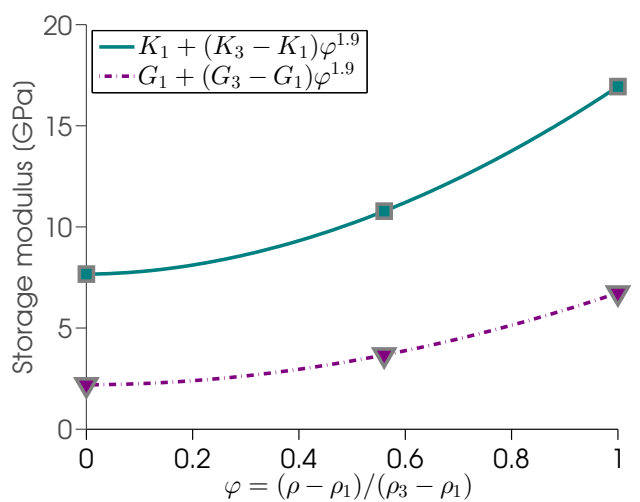

(a) (a)

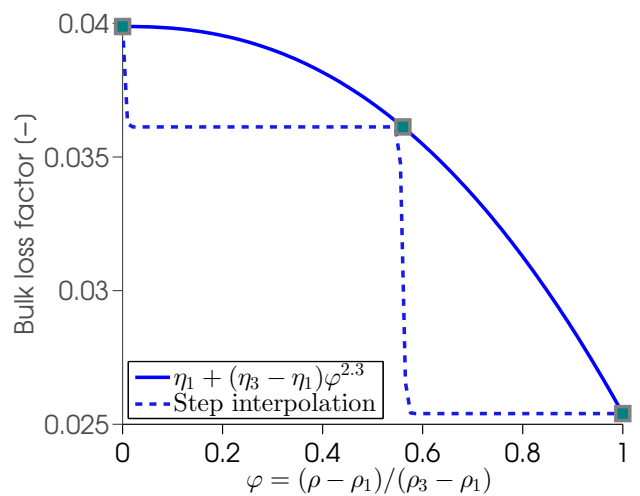

(c) $(\mathrm{c})$

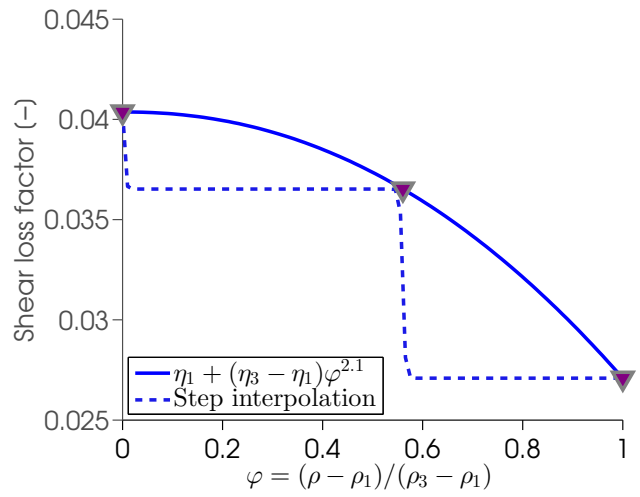

(e) (e)

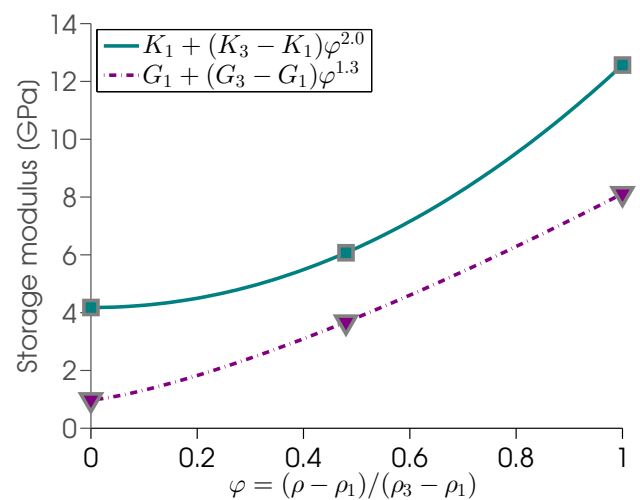

(b) (b)

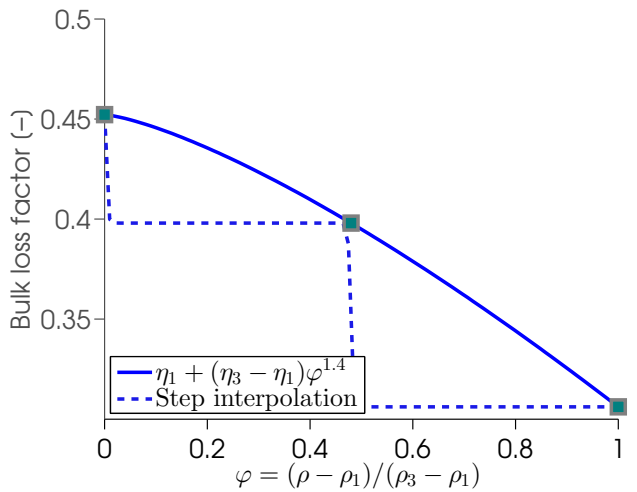

(d) (d)

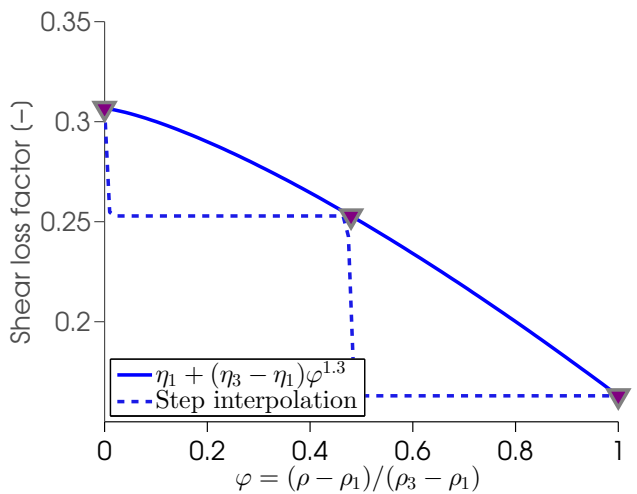

(f) (f)

Fig. 7: (a), (c), and (e) show material interpolations between microstructures 1-3 in Fig. 5(a). (b), (d), and (f) show material interpolations between microstructures 1-3 in Fig. 6(a). (a-b) show the storage moduli interpolations, and (c-f) show the loss factor interpolation.

and we subtract this value from $f_{i}$, because we want the function to take the value 0 at $\varphi=1$, and redefine $f_{i}$ :

$$
f_{i}(\varphi)=\tanh \left(\beta \varphi_{i}-\beta \varphi\right)-\tanh \left(\beta \varphi_{i}-\beta\right)
$$

which will take the value

$$
f_{i}(0)=\tanh \left(\beta \varphi_{i}\right)-\tanh \left(\beta \varphi_{i}-\beta\right),
$$

at $\varphi=0$. We want the function to take the value 1 at $\varphi=0$, and therefore we again redefine $f_{i}$ :

$$
f_{i}(\varphi)=\frac{\tanh \left(\beta \varphi_{i}-\beta \varphi\right)-\tanh \left(\beta \varphi_{i}-\beta\right)}{\tanh \left(\beta \varphi_{i}\right)-\tanh \left(\beta \varphi_{i}-\beta\right)} .
$$




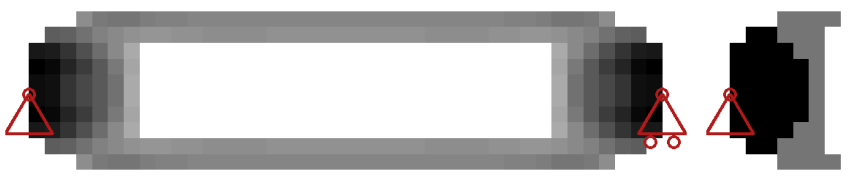

(a) (a) $\zeta_{1}=0.019$

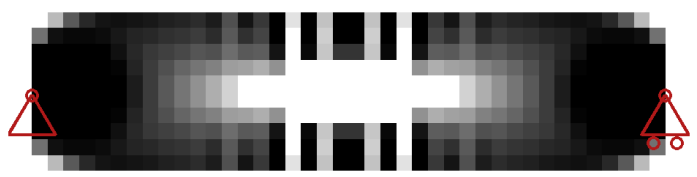

(c) $\left(\right.$ c) $\zeta_{1}=0.131$

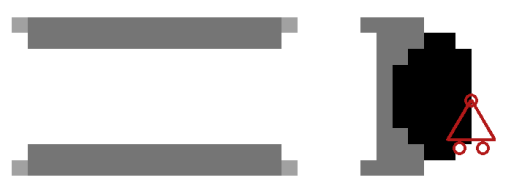

(b) (b) $\zeta_{1}=0.019$

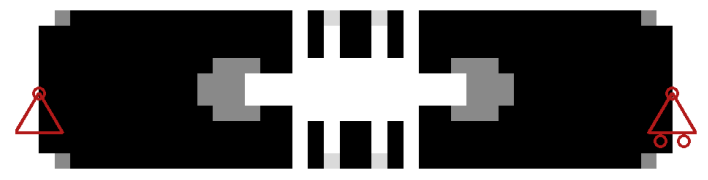

(d) (d) $\zeta_{1}=0.128$

Fig. 8: Optimized beams, where white indicates microstructure 1 and black indicates microstructure 3. Gray corresponds to intermediate microstructures. (a) shows the design obtained using the power law interpolation for S1 microstructures, and (b) shows the final design after continuing optimization with Heaviside interpolation. (c) shows the design obtained using the power law interpolation for S2 microstructures, and (d) shows the final design after continuing the optimization with Heaviside interpolation.

By creating a weighted sum of these functions with step values $\varphi_{i}$ corresponding to the material densities of the microstructures, a multiple step function between the loss factors of the microstructures can be obtained. This can be expressed as:

$$
\eta=\eta_{n}+\sum_{i=1}^{n-1}\left(\eta_{i}-\eta_{i+1}\right) f_{i},
$$

where $\eta_{i}$ is loss factor for microstructure $i$, and $n$ is the number of microstructures.

The step function interpolation is first introduced after a good design has been obtained, and, therefore, the parameter controlling the steepness of the steps is set to a constant high value $\beta=200$.

\section{Macroscopic results}

Consider the earlier described simply supported beam. If there is no constraint on the lowest eigenvalue, the optimized structure would be one purely consisting of microstructure 1. With S1 as the soft phase, the stiffest microstructure has $\zeta_{1}=0.013$ and $\operatorname{Re}\left(\omega_{1}\right)=1219 \mathrm{~Hz}$. Thus, for the optimized beam we allow a slightly lower first eigenfrequency of $0.8 \cdot 1219 \mathrm{~Hz}=975 \mathrm{~Hz}$, with the aim of achieving a higher structural damping.

The result of the first part of the optimization, using only the power law interpolations for the S1 microstructures from Fig. 7, is shown in Fig. 8(a). The optimized beam has $\zeta_{1}=0.019$. Continuing with the step function interpolation results in the design in Fig. 8(b). A design with three discrete density values is nearly obtained. This beam also has a damping ratio that is only one percent lower than the beam in Fig. $8(\mathrm{a})$.

Now we run the same optimization, that is with the same eigenvalue constraint, but with the material interpolations for S2 from Fig. 7. The beam designs with the power law and the step function interpolation can be seen in Fig. 8(c) and (d), respectively. The damping ratios for the two designs are 0.131 and 0.128.

The large difference in damping ratios between the beam with $\mathrm{S} 1$ and $\mathrm{S} 2$, is to be expected since the base material properties are very different. However, it is also clear that the beam with $\mathrm{S} 2$ will contain much more titanium in order to achieve the eigenvalue constraint. This is very clearly seen in Fig. 9, where the beams from Fig. 8(b,d) are shown with the full microstructural pattern.

Homogenization theory, which is used to compute the macroscopic material properties, assumes that there is a clear separation of scales. But as we discuss, the manufacturing requirements will impose a minimum length scale - severely restricting how many unit cell repetitions the part can contain; especially when the part contains a varying microstructure (as in the examples presented here). That said, based on e.g. the recent numerical results presented by Alexandersen and Lazarov (2015), we have reason to believe that a slowly varying microstructure give macroscopic properties close to the ones predicted by homogenization theory.

Finally, it should be remarked that only the first eigenfrequency has been included in the optimizations here, even though the min-max formulation in Eq. (1) allows for a range of eigenfrequency. And the approach 


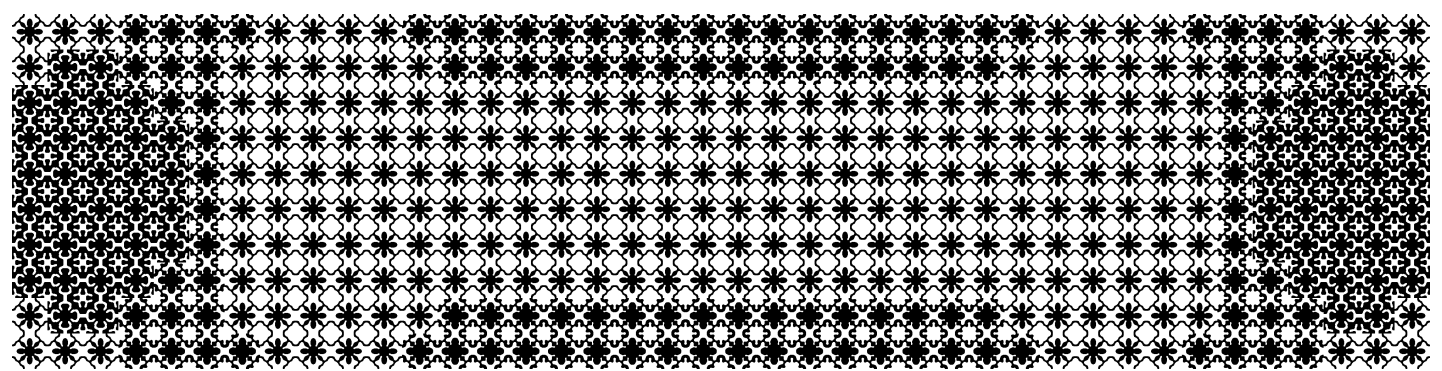

(a) (a)

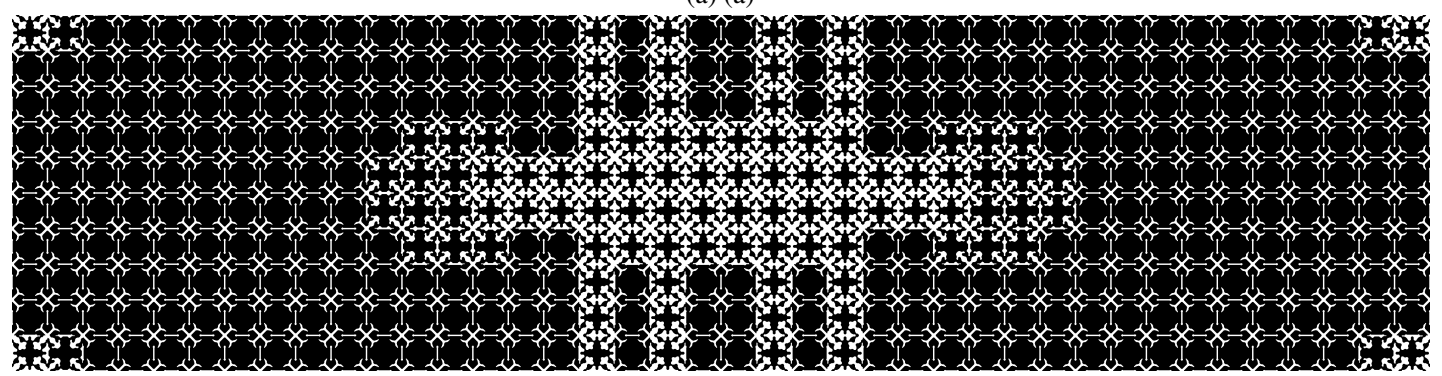

(b) (b)

Fig. 9: Optimized beams from Fig. 8(b) and (d) with microstructure. (a) S1 microstructure, (b) S2 microstructure. Black indicates stiff material phase and white indicates soft material phase.

does work equally well with a range of eigenfrequencies, but in most cases the structural damping of the first one or two eigenfrequencies will drive the optimization.

\section{Conclusion}

The presented simple multiscale formulation consists of a two step optimization approach, where one first uses topology optimization to obtain a range of manufacturable microstructures, and, thereafter, use a material interpolation based on the homogenized properties in the macroscopic problem. A multiple step function is introduced to obtain optimized structural damping designs with a discrete set of microstructures.

The approach makes it computationally feasible to maximize the structural damping ratio of a component with fine details. Thus, it can also be extended to three-dimensional (3D) problems without being computationally exhaustive. Furthermore, it assures the component is manufacturable, because the microstructural design guarantees the connectivity of the stiff phase, and the suggested material interpolation guides the design to a discrete set of microstructures.

None of the presented designs have been manufactured, but it will be natural to manufacture and test designs in conjunction with applying the approach for 3D problems. The approach of printing a stiff phase (e.g. in titanium) and infusing it with a soft phase is expensive, and is most attractive for high-end, low volume products. Possible applications could be aerospace components, high-end sports parts (e.g. racing bike saddle post), and high quality audio equipment, such as a tone arm (basically a simply supported beam).

\section{References}

Alexandersen J, Lazarov BS (2015) Topology optimisation of manufacturable microstructural details without length scale separation using a spectral coarse basis preconditioner. Comput Method Appl Mech Eng 290:156-182

Andreasen CS, Sigmund O (2012) Multiscale modeling and topology optimization of poroelastic actuators. Smart Mater Struct 21(6)

Andreasen CS, Andreassen E, Jensen JS, Sigmund O (2014) On the realization of the bulk modulus bounds for two-phase viscoelastic composites. J Mech Phys Solids 63(1):228-241 
Andreassen E, Andreasen CS (2014) How to determine composite material properties using numerical homogenization. Comput Mat Sci 83:488-495, DOI 10.1016/j.commatsci.2013.09.006

Barbarosie C, Toader AM (2012) Optimization of bodies with locally periodic microstructure. Mech Adv Mater Struc 19(4):290-301

Bendsøe M, Guedes J, Haber R, Pedersen P, Taylor J (1994) An analytical model to predict optimal material properties in the context of optimal structural design. J Appl Mech-T ASME 61(4):930-937

Coelho PG, Fernandes PR, Guedes JM, Rodrigues HC (2008) A hierarchical model for concurrent material and topology optimisation of three-dimensional structures. Struct Multidisc Optim 35(2):107-115

Coelho PG, Cardoso JB, Fernandes PR, Rodrigues HC (2011) Parallel computing techniques applied to the simultaneous design of structure and material. Adv Eng Softw 42(5):219-227

El-Sabbagh A, Baz A (2014) Topology optimization of unconstrained damping treatments for plates. Eng Optim 46(9):1153-1168

Guedes JM, Kikuchi N (1990) Preprocessing and postprocessing for materials based on the homogenization method with adaptive finite element methods. Comput Method Appl Mech Eng 83(2):143-198

Guest J, Prevost J, Belytschko T (2004) Achieving minimum length scale in topology optimization using nodal design variables and projection functions. Int J Numer Meth Eng 61(2):238-254

Hassani B, Hinton E (1998) A review of homogenization and topology optimization I - homogenization theory for media with periodic structure. Computers \& Structures 69(6):707-717

Hughes TJR (1980) Generalization of selective integration procedures to anisotropic and nonlinear media. Int J Numer Meth Eng 15(9):1413-1418

Khanoki SA, Pasini D (2012) Multiscale design and multiobjective optimization of orthopedic hip implants with functionally graded cellular material. J Biomech Eng-T ASME 134(3):031,004

Kim SY, Mechefske CK, Kim IY (2013) Optimal damping layout in a shell structure using topology optimization. J Sound Vib 332(12):2873-2883

Ling Z, Ronglu X, Yi W, El-Sabbagh A (2011) Topology optimization of constrained layer damping on plates using method of moving asymptote (mma) approach. Shock Vib 18(1-2):221-244

Rodrigues H, Guedes JM, Bendsoe MP (2002) Hierarchical optimization of material and structure. Struct Multidisc Optim 24(1):1-10

Stegmann J, Lund E (2005) Discrete material optimization of general composite shell structures. Int J Numer Meth Eng 62(14):2009-2027

Svanberg K (1987) Methods of moving asymptotes - a new method for structural optimization. Int J Numer Meth Eng 24(2):359-373

Theocaris PS, Stavroulakis GE (1999) Optimal material design in composites: An iterative approach based on homogenized cells. Comput Method Appl Mech Eng 169(1-2):31-42

Wang F, Lazarov BS, Sigmund O (2011) On projection methods, convergence and robust formulations in topology optimization. Struct Multidisc Optim 43(6):767-784

Xu S, Cai Y, Cheng G (2010) Volume preserving nonlinear density filter based on heaviside functions. Struct Multidisc Optim 41(4):495-505 